\title{
ENFERMEDAD AUTOINMUNE SISTÉMICA EN PACIENTES CON UVEÍTIS
}

\section{SYSTEMIC AUTOIMMUNE DISEASE IN PATIENTS WITH UVEITIS}

\author{
CARBONE J ${ }^{1}$, SARMIENTO E ${ }^{2}$, MICHELOUD D ${ }^{2}$, RODRÍGUEZ-MAHOU $\mathrm{M}^{3}$, \\ RODRÍGUEZ-MOLINA JJ ${ }^{4}$, COBO R ${ }^{5}$, FERNÁNDEZ-CRUZ E ${ }^{1}$
}

\section{RESUMEN}

Objetivo: La realización de un estudio descriptivo en pacientes con uveítis para establecer la frecuencia de enfermedad autoimmune sistémica asociada. Métodos: Se incluyeron en el estudio 64 pacientes con uveítis. Ninguno de los pacientes estudiados tenia una enfermedad autoinmune sistémica conocida antes del diagnóstico de uveítis. A todos los pacientes se les realizó un protocolo diagnóstico que incluyó las siguientes pruebas inmunológicas: inmunoglobulinas séricas, factores del complemento, inmunocomplejos circulantes (ICC), anticuerpos antinucleares (ANA), anticuerpos anticitoplasma de neutrófilos (ANCA), anticuerpos anticardiolipina (ACA) y antígenos del complejo mayor de histocompatibilidad.

Resultados: En once casos $(17,2 \%)$ se objetivó asociación con un proceso autoinmune subclínico caracterizado por la positividad de autoanticuerpos (ANA, ANCA o ACA) en presencia de consumo de complemento, hipergammaglobulinemia o ICC ele-

\begin{abstract}
Objective: A descriptive study was conducted on patients with uveitis to determine the frequency of associated autoimmune systemic diseases.

Methods: 64 patients with uveitis were studied. The patients were not known to have an underlying autoimmune systemic disease prior to the diagnosis of uveitis. All patients had the following immunological tests performed: serum immunoglobulins, complement components, circulating immune complexes (CIC), antinuclear antibodies (ANA), antineutrophil cytoplasmic antibodies (ANCA), anticardiolipin antibodies (ACA) and major histocompatibilty complex antigens.

Results: A relationship with a sub-clinical autoimmune systemic disorder could be presumed in eleven cases $(17.2 \%)$. This was defined by positive autoantibodies (ANA, ANCA or ACA) in the presence of complement consumption, hyper-gammaglobulinemia or increased CIC without clinical criteria of a defined autoimmune disease. A definite
\end{abstract}

Recibido: 29/4/05. Aceptado: 17/4/06.

Unidad de Inmunología Clínica. Servicio de Inmunología. Hospital General Universitario Gregorio Marañón. Madrid. España.

1 Doctor en Medicina.

2 Licenciado en Medicina.

3 Doctor en Ciencias Biológicas

4 Licenciado en Ciencias Biológicas.

5 Doctor en Medicina. Servicio de Oftalmología.

Presentado parcialmente en el XXVII Congreso de la Sociedad Española de Inmunología (Madrid 2001) y en el $3^{\text {rd }}$ International Congress on Autoimmunity (Geneva 2002).

Correspondencia:

Javier Carbone

Unidad de Inmunología Clínica. Servicio de Inmunología

Hospital General Universitario Gregorio Marañón

C/. Doctor Esquerdo, 46

28007 Madrid

España

E-mail: carbone@teleline.es 
vados, sin que los pacientes cumpliesen criterios clínicos de una enfermedad autoinmune. Se encontró una asociación definitiva con enfermedad autoimmune sistémica en cuatro pacientes $(6,25 \%)$. Las enfermedades autoinmunes observadas fueron el síndrome de Sjögren $(n=2,3,13 \%)$, síndrome antifosfolípido asociado a enfermedad lupus like $(\mathrm{n}=1,1,6 \%)$ y vasculitis sistémica $(\mathrm{n}=1,1,6 \%)$. También se observó un grupo de pacientes con enfermedad lupus like $(n=4,6,25 \%)$.

Conclusión: En una proporción de pacientes con uveítis puede existir un proceso autoimmune sistémico subyacente.

Palabras clave: Uveítis, enfermedad autoinmune sistémica, lupus. association with systemic autoimmune disease was defined in four patients $(6.25 \%)$. The observed autoimmune systemic diseases were Sjögren's syndrome $(n=2,3.13 \%)$, anti-phospholipid syndrome associated with lupus-like disease ( $\mathrm{n}=1,1.6 \%)$, and systemic vasculitis $(n=1,1.6 \%)$. Lupus-like disease $(\mathrm{n}=4,6.25 \%)$ was also observed.

Conclusion: In a significant proportion of patients with uveitis an autoimmune systemic disorder may be present and should be looked for (Arch Soc Esp Oftalmol 2006; 81: 193-198).

Key words: Uveitis, systemic autoimmune disease, lupus.

\section{INTRODUCCIÓN}

Las uveítis son un grupo de enfermedades inflamatorias oculares que afectan las capas medias del ojo: iris, cuerpo ciliar y coroides $(1,2)$. Aunque la etiología suele ser desconocida en la mayoría de los casos, algunos pacientes pueden tener una enfermedad sistémica subyacente incluyendo enfermedades infecciosas y autoinmunes (3-5). En el presente trabajo se investiga la prevalencia de enfermedad autoinmune sistémica subyacente en pacientes con uveítis, tras la realización de un estudio inmunológico.

\section{SUJETOS, MATERIAL Y MÉTODOS}

Se estudiaron retrospectivamente 64 pacientes diagnosticados de uveítis procedentes del Servicio de Oftalmología y otros Servicios del Hospital. Las características demográficas y clínicas de los pacientes se presentan en la tabla I. En todos los casos el diagnóstico de uveítis se estableció en los servicios de referencia en base a la historia clínica, la exploración oftalmológica y pruebas complementarias. No se incluyeron en el estudio pacientes con enfermedad autoinmune sistémica previamente conocida. Se estudiaron las alteraciones inmunológicas, clínicas y de laboratorio que presentaban los pacientes en asociación con la uveítis.
Tabla I. Características demográficas y clínicas de 64 pacientes con uveítis

\begin{tabular}{lc}
\hline Parámetro & Número $(\%)$ \\
\hline Sexo & \\
Hombres & $33(52)$ \\
Mujeres & $31(48)$ \\
Edad, años. Media (intervalo) & $42(19-78)$ \\
Localización de la uveítis & \\
Anterior & $38(59,4)$ \\
Posterior & $14(21.9)$ \\
Intermedia & $4(6,3)$ \\
Panuveítis & $8(12,5)$ \\
Unilateral & $59(92,2)$ \\
Bilateral & $5(7,8)$ \\
Número de episodios & \\
Uno & $46(71,9)$ \\
Dos & $15(23,4)$ \\
> de dos & $3(4,7)$ \\
Tratamiento & \\
Corticoides tópicos & $24(37,5)$ \\
Corticoides sistémicos & $35(54,7)$ \\
Ciclosporina & $24(37,5)$ \\
Duración del tratamiento & \\
< de 3 meses & $54(84,4)$ \\
> de 3 meses & $10(15,6)$ \\
Complicación oftalmológica asociada & $6(9,4)$ \\
Hemorragia vítrea & $10(15,6)$ \\
Sinequias & $2(3,1)$ \\
Edema de papila & $3(4,7)$ \\
Papilitis & $3(4,7)$ \\
Desprendimiento de retina & $2(3,1)$ \\
Trombosis de retina & $1(1,6)$ \\
Atrofia óptica & $1(1,6)$ \\
Vasculitis & \\
\hline \hline
\end{tabular}




\section{Estudio de autoinmunidad}

La detección de anticuerpos antinucleares (ANA) se realizó mediante la técnica de inmunofluorescencia indirecta (IFI) sobre células HEp-2 (BION, Alemania); los anticuerpos anti-ADN de doble cadena, y los anticuerpos anti-antígenos nucleares extraíbles (anti-ENA) se estudiaron mediante técnica de ELISA; los anticuerpos anti-citoplasma de neutrófilos (ANCA) se estudiaron mediante técnica de IFI utilizando neutrófilos fijados en etanol y posteriormente mediante técnica de ELISA de anticuerpos anti-mieloperoxidasa (anti-MPO) o anti-proteinasa3 (anti-PR3); los anticuerpos anticardiolipina (ACA de los isotipos $\operatorname{IgG}$ e $\operatorname{IgM}$ ) se realizaron mediante técnica de ELISA dependiente de beta-2-glicoproteína-I (Orgentec Diagnostika GmbH, Mainz, Alemania); el factor reumatoide (FR) se estudió mediante técnica de nefelometría (Beckman Coulter Izasa, California, Estados Unidos).

\section{Estudio de inmunidad humoral}

Los niveles séricos de inmunoglobulinas ( $\mathrm{IgG}$, $\operatorname{Ig} \mathrm{A}, \operatorname{IgM})$, y de los factores del complemento (C3, C4 y Factor B) se determinaron mediante técnica de nefelometría (Beckman Coulter Izasa, California, Estados Unidos); la actividad hemolítica total del complemento (CH100) se estudió mediante inmunodifusión radial (Binding Site Limited, Birmingham, Inglaterra) y los inmunocomplejos circulantes (ICC) mediante ensayo nefelométrico dependiente de C1q (Dade Behring Nephelometer Analyser II, Marburg, Alemania y Quidel, San Diego California, Estados Unidos).

\section{Estudio de antígenos de histocompatibilidad}

El estudio de antígenos del sistema HLA de clase I y II se realizó mediante técnica serológica de microlinfocitotoxidad.

\section{Otras pruebas diagnósticas}

Recuento sanguíneo completo, examen completo de orina, bioquímica básica y serología a lúes. Otras pruebas complementarias se realizaron a los pacientes que presentaban datos clínicos o de labo- ratorio de alta sospecha de una conectivopatía sistémica.

En los pacientes que presentaron positividad de autoanticuerpos (especialmente ANA, ANCA o ACA), evidencia de consumo de complemento (cifras de $\mathrm{C} 3, \mathrm{C} 4$ o $\mathrm{CH} 100$ bajas) e hipergammaglobulinemia o ICC elevados, en ausencia de criterios clínicos de conectivopatía, se consideró la presencia de un proceso autoinmune subclínico (6).

Los pacientes que reunían 4 criterios de la Academia Americana de Reumatología para la clasificación de Lupus Eritematoso Sistémico (LES) eran clasificados como tal (7). Los pacientes que reunían 3 de los criterios antes mencionados eran considerados Enfermedad Lupus Like (ELL). Otras enfermedades autoinmunes sistémicas se diagnosticaron según los criterios vigentes en el momento de la realización del protocolo diagnóstico.

\section{RESULTADOS}

La mayoría de los pacientes del estudio (61\%) no presentaron datos clínicos de enfermedad autoinmune sistémica. En la tabla II se muestran los síntomas y signos prevalentes en los pacientes. La artritis, úlceras orales y linfopenia fueron las alteraciones clínicas más frecuentes. La distribución global de las alteraciones inmunológicas detectadas se presentan en la tabla III. La asociación con antígenos HLA de clase I y II se muestra en la tabla IV. La uveítis anterior asociada a HLAB27(+) se observó en 8 pacientes $(12,5 \%)$. Un paciente tuvo presunción diagnóstica de Síndrome de Vogt-Koyanagui-Harada y otro de retinocoroidopatía de birdshot.

Tabla II. Síntomas y signos asociados en 64 pacientes con uveítis

$\begin{array}{lrr}\text { Síntomas y signos asociados } & \text { Número }(\%) \\ \text { Ninguno } & 39 & (60,9) \\ \text { Artritis } & 6 & (9,4) \\ \text { Sicca } & 3 & (4,7) \\ \text { Úlceras orales } & 7 & (10,9) \\ \text { Rash } & 2 & (3,1) \\ \text { Sacroileitis } & 2 & (3,1) \\ \text { Hipoacusia y vitiligo } & 2 & (3,1) \\ \text { Aborto } & 1 & (1,6) \\ \text { Anemia } & 4 & (6,3) \\ \text { Linfopenia } & 13 & (20,3) \\ \text { Trombopenia } & 3 & (4,7) \\ \end{array}$


Tabla III. Alteraciones inmunológicas en 64 pacientes con uveítis

\begin{tabular}{lrr}
\hline Parámetros & Número $(\%)$ \\
\hline Hipergammaglobulinemia & & \\
IgA & 3 & $(4,7)$ \\
IgG & 7 & $(10,9)$ \\
IgM & 5 & $(7,8)$ \\
Hipogammaglobulinemia & 6 & $(9,4)$ \\
IgA & 6 & $(9,4)$ \\
IgG & 3 & $(4,7)$ \\
IgM & 6 & $(9,4)$ \\
Hipocomplementemia & 14 & $(21,9)$ \\
C3 & 17 & $(26,6)$ \\
C4 & $27(42,2)$ \\
CH100 bajo & \\
Inmunocomplejos circulantes altos & $14(21,9)$ \\
Autoanticuerpos & 5 & $(7,8)$ \\
Anticuerpos antinucleares (Título 1/40-1/320) & $7(10,9)$ \\
Anticuerpos anticitoplasma de neutrofilos & $8(12,5)$ \\
Anticuerpos anticardiolipina IgG & 4 & $(6,3)$ \\
Anticuerpos anticardiolipina IgM & \\
Factor Reumatoide & \\
\hline
\end{tabular}

Once pacientes $(17,2 \%)$ tenían positividad de autoanticuerpos junto con evidencia de consumo de complemento, hipergammaglobulinemia o ICC elevados, sin reunir criterios clínicos de clasificación de conectivopatía. La presencia de estas alteraciones inmunológicas no tuvo una asociación significativa con parámetros clínicos como la localización de la uveítis, el tiempo de tratamiento o la aparición de complicaciones oftalmológicas (prueba de chicuadrado, $p>0,05)$.

Se detectó una asociación definitiva con un proceso autoinmune sistémico en cuatro casos $(6,25 \%)$. La edad en los pacientes con conectivopatía sistémica fue similar al resto de pacientes. Sin embargo hubo una afectación significativamente mayor de mujeres (prueba exacta de Fischer, $p<0,05)$. Tampoco en este grupo encontramos diferencias significativas en cuando al curso clínico de la uveítis. Las enfermedades autoinmunes observadas fueron síndrome antifosfolípido asociado a ELL $(\mathrm{n}=1,1,56 \%)$, síndrome de Sjögren $(\mathrm{n}=2,3,12 \%)$ y vasculitis sistémica $(n=1,1,56 \%)$. La paciente diagnosticada de síndrome antifosfolípido inició tratamiento profiláctico primario de trombosis con ácido acetil salicílico a baja dosis y la paciente que desarrolló vasculitis sistémica, posteriormente al diagnóstico de uveítis, inició tratamiento con ciclofosfamida y corticoides.

Otros cuatro pacientes fueron diagnosticados de ELL (6,25\%). En la tabla V se muestran las alteraciones clínicas e inmunológicas observadas en los cuatro pacientes con enfermedad autoinmune sistémica y en los cuatro pacientes con ELL. Los cuatro pacientes que asociaron enfermedad autoinmune sistémica habían tenido una uveítis anterior. Dos de los cuatro pacientes con ELL tuvieron una uveítis anterior y los otros dos una uveítis intermedia. Dos pacientes presentaron ELL asociada a sacroileitis, uno de los cuales tenía el antígeno de clase I HLA-B27 (tabla V).

\section{DISCUSIÓN}

El ojo, y especialmente los tejidos más vascularizados como la conjuntiva y la uvea, pueden ser diana de reacciones inmunopatológicas. La uveítis puede ser la primera manifestación de una enfermedad autoimmune sistémica, y preceder a la aparición del proceso sistémico (1). La uveítis se puede asociar con procesos de base inmunológica como la enfermedad de Behcet, sarcoidosis, artritis reumatoide juvenil, esclerosis múltiple, lupus eritematoso y con el grupo de espondiloartropatías asociadas al antígeno HLA B27 (2-5).

En el presente trabajo se estudia la prevalencia de enfermedad autoinmune sistémica en pacientes remitidos para evaluación inmunológica con diagnóstico de uveítis sin enfermedad sistémica conocida. Se debe tener en cuenta que se trata de un grupo de pacientes seleccionado por las evaluaciones clínicas realizadas previamente en los servicios de referencia.

Tabla IV. Antígenos HLA de clase I y II asociados en 64 pacientes con uveítis

\begin{tabular}{|c|c|c|}
\hline Diagnóstico & Antígeno & Número (\%) \\
\hline Uveítis Anterior & HLA B27 & $8(12,5)$ \\
\hline Uveítis Posterior & HLA DR52 & $2(3,1)$ \\
\hline Síndrome de Vogt-Koyanagui-Harada & HLA DR53 & $1 \quad(1,6)$ \\
\hline Retinocoroidopatía en perdigonada & HLA A29 & $1 \quad(1,6)$ \\
\hline
\end{tabular}


Tabla V. Características clínicas e inmunológicas de 8 pacientes con uveítis que asociaron enfermedad autoinmune sistémica o ELL

\begin{tabular}{|c|c|c|c|c|}
\hline Paciente & Enfermedad asociada & Clínica asociada & Inmunidad humoral & Autoinmunidad \\
\hline $1^{*}$ & SAAF+ELL & $\mathrm{AB}$, artritis, $\mathrm{UO}$, anemia & C3, C4, CH100, ICC & $\begin{array}{l}\text { ANA } 1 / 40 \text { (homogeneo) } \\
\text { ACA IgG e IgM }\end{array}$ \\
\hline $2^{*}$ & Síndrome de Sjögren & Sicca, linfopenia & $\mathrm{C} 3, \mathrm{C} 4, \mathrm{CH} 100, \mathrm{ICC}, \mathrm{IgG}$ & $\begin{array}{l}\text { ANA } 1 / 160 \\
\quad(\text { Granular-NuMa) }\end{array}$ \\
\hline $3 *$ & Síndrome de Sjögren & $\begin{array}{l}\text { Sicca, artralgias, RF, UO, anemia, } \\
\text { linfopenia }\end{array}$ & $\mathrm{C} 4$ & $\begin{array}{l}\text { ANA } 1 / 160 \text { (homogeneo). } \\
\text { ACA IgG e IgM }\end{array}$ \\
\hline $4 *$ & Vasculitis Sistémica & Vasculitis renal, sinusitis & $\mathrm{C} 4$ & $\begin{array}{l}\text { P-ANCA > 1/20. } \\
\text { Anti-MPO } 31 \mathrm{U} / \mathrm{ml}\end{array}$ \\
\hline $5 * *$ & ELL & UO, vasculitis retina & $\mathrm{C} 3, \mathrm{C} 4, \mathrm{ICC}$ & $\begin{array}{l}\text { ANA } 1 / 40 \text { (homogeneo), } \\
\text { ACA IgM }\end{array}$ \\
\hline $6^{*}$ & ELL & UO, artritis, LR, linfopenia & CH100, ICC & ACA IgM \\
\hline $7 *$ & ELL & $\begin{array}{l}\text { Artritis, sacroileitis anemia, } \\
\text { linfopenia, }\end{array}$ & C4, HLA-B27(+) & $\begin{array}{l}\text { ANA 1/160 (Mixto). } \\
\text { X-ANCA } 1 / 20\end{array}$ \\
\hline $8 * *$ & ELL & Artritis, sacroileitis, linfopenia & C4, ICC, HLA-B27(-), & $\begin{array}{l}\text { ANA } 1 / 40 \text { (homogeneo). } \\
\text { ACA IgG e IgM }\end{array}$ \\
\hline
\end{tabular}

* Uveítis anterior; ** Uveítis intermedia; $\mathrm{AB}=$ abortos, ELL= Enfermedad lupus like, C3= C3 bajo, C4= C4 bajo, CH100= CH100 bajo, ICC= ICC elevados, $\mathrm{IgG}=$ hipergammaglobulinemia $\mathrm{IgG}, \mathrm{LR}=$ livedo reticularis, $\mathrm{RF}=$ rash fotosensible, $\mathrm{SAAF}=\mathrm{Síndrome}$ de anticuerpos antifosfolípidos, $\mathrm{UO}=$ úlceras orales.

Un subgrupo de pacientes presentó un proceso autoinmune sistémico. En otro subgrupo de pacientes se observaron datos clínicos de ELL. La presencia de uveítis anterior e intermedia en los pacientes con ELL es interesante ya que en pacientes que cumplen criterios de lupus eritematoso sistémico se describe más frecuentemente la uveítis posterior $(1,8)$. Igualmente interesante ha sido la asociación de ELL y sacroileitis en dos pacientes con uveítis, por ser poco habitual (9).

Por otro lado, se ha observado un subgrupo de pacientes con uveítis que cursan con autoanticuerpos y datos de consumo de complemento, hipergammaglobulimenia o ICC aumentados sin tener criterios de clasificación de una enfermedad autoinmune conocida. Estas alteraciones inmunológicas no parecen haber influido en el curso clínico de la uveítis y desconocemos si puedan haber participado en la patogenia de la uveítis. Sin embargo, estos pacientes están siendo evaluados prospectivamente para descartar la evolución a un proceso autoinmune con repercusión clínica sistémica. En trabajos previos, realizados en pacientes con otras patologías de base inmunológica, alteraciones inmunológicas similares han precedido en meses o años a la aparición de conectivopatía en un pequeño porcentaje de pacientes con dichas alteraciones $(6,10)$.

Un aspecto práctico del presente trabajo está en relación con la justificación de la realización de pruebas complementarias inmunológicas en pacien- tes con uveítis. Se ha sugerido que la realización de pruebas adicionales debe indicarse cuando la inflamación es moderada a severa, bilateral, recurrente, granulomatosa, o está asociada con síntomas sistémicos que sugieran una enfermedad subyacente que puede ser causa conocida de uveítis (11). En el presente trabajo un $39 \%$ de los pacientes tenían síntomas extraoculares que podrían ser indicativos de proceso autoinmune sistémico. Aunque el porcentaje de pacientes con proceso autoinmune sistémico asociado a uveítis ha sido bajo, se detectó un subgrupo de pacientes con ELL y cerca del $20 \%$ de los pacientes tenían alteraciones inmunológicas compatibles con un proceso autoinmune subclínico. Por ello, entendemos que un estudio inmunológico como el realizado podría ser útil como parte del proceso de descarte de procesos autoinmunes sistémicos asociados al cuadro de uveítis, especialmente en los pacientes que presenten síntomas extraoculares.

\section{AGRADECIMIENTOS}

A los Dres. Silvia Sánchez-Ramón, Elena Seoane, Jose Luis Ruiz-Tíscar y Jesús Bermejo del Servicio de Inmunología por su participación al atender pacientes en la Consulta de Inmunología. A los facultativos especialistas del Servicio de Oftalmología y otros Servicios del Hospital que refirieron 
pacientes para la realización del estudio inmunológico. Al Dr. José Luis Vicario del Centro de Transfusiones de la Comunidad de Madrid, por la realización de los estudios de antígenos de histocompatibilidad de clase I y II.

\section{BIBLIOGRAFÍA}

1. Whitcup SL, Nussenblatt RB. Immunologic Ocular Disease. In: Rich RR. Clinical Immunology, Principles and Practice. St Louis: Mosby; 1996; II: 1579-1591.

2. Durrani OM, Meads CA, Murray PI. Uveitis: a potentially blinding disease. Ophthalmologica 2004; 218: 223-236.

3. Tay-Kearney ML, Schwam BL, Lowder C, Dunn JP, Meisler DM, Vitale S, et al. Clinical features and associated systemic diseases of HLA-B27 uveitis. Am J Ophthalmol 1996; 121: 47-56.

4. Rothova A, Buitenhuis HJ, Meenken C, Brinkman CJ, Linssen A, Alberts $C$, et al. Uveitis and systemic disease. Br J Ophthalmol 1992; 76: 137-141.

5. McCluskey P, Powell RJ. The eye in systemic inflammatory diseases. Lancet 2004; 364: 2125-2133.
6. Carbone J, Sanchez-Ramon S, Cobo-Soriano R, Seoane E, Aparicio MJ, Ruiz-Tiscar JL, et al. Antiphospholipid antibodies: a risk factor for occlusive retinal vascular disorders. Comparison with ocular inflammatory diseases. $J$ Rheumatol 2001; 28: 2437-2441.

7. Tan EM, Cohen AS, Fries JF, Masi AT, McShane DJ, Rothfield NF, et al. The 1982 revised criteria for the classification of systemic lupus erythematosus. Arthritis Rheum 1982; 25: 1271-1277.

8. Cervera R, Khamashta MA, Font J, Sebastiani GD, Gil A, Lavilla $P$, et al. Morbidity and mortality in systemic lupus erythematosus during a 5-year period. A multicenter prospective study of 1,000 patients. European Working Party on Systemic Lupus Erythematosus. Medicine (Baltimore) 1999; 78: 167-175.

9. Kohli M, Bennett RM. Sacroileitis in systemic lupus erythematosus. J Rheumatol 1994; 21: 170-171.

10. Carbone J, Sarmiento E, Segovia P, Rodríguez-Mahou M, Rodríguez-Molina JJ, Fernández-Cruz. E. Anticuerpos antifosfolípidos y evolución a conectivopatía: Estudio de seguimiento de mujeres con abortos de repetición. Med Clin (Barc) 2002; 119: 681-685.

11. Chipont Benabent E, Cunningham ET Jr. Manejo del paciente con uveítis anterior aguda. Arch Soc Esp Oftalmol 2002; 77: 183-193. 